short courses of AST: 0 vs 3 vs 6 months, 3 vs 8 months, or 0 vs 6 months in trials from Australasia, Canada and the US, respectively. Men aged $\geq 65$ years who received 6 months of AST experienced a shorter time to fatal MI than those $\geq 65$ years who did not receive AST treatment. Follow up of men $\leq 65$ years was insufficient to determine whether these younger men were affected similarly. In addition, the study had limited power to measure whether 3 months of AST treatment significantly shortened time to fatal MI compared with no AST treatment.

The authors suggest that men $\geq 65$ years with prostate cancer undergo a cardiovascular evaluation before commencing AST and RT treatment so that their risk of MI can be assessed and if necessary treated appropriately before initiating AST.

Original article D'Amico AV et al. (2007) Influence of androgen suppression therapy for prostate cancer on the frequency and timing of fatal myocardial infarctions. J Clin Oncol 25:2420-2425

\section{Transobturator routes for suburethral tape placement not superior to retropubic insertion}

Outside-in transobturator tape (TOT) and inside-out tension-free vaginal obturator tape (TVTO) insertion were introduced to reduce the risk of complications associated with tensionfree vaginal tape (TVT) for treatment of female urinary stress incontinence. Good initial results led to widespread use before efficacy and safety data were available. In this meta-analysis and review, Latthe et al. assess the respective cure and complication rates.

The investigators searched online for reports published between January 1966 and September 2006. They did further manual searches of bibliographies in identified articles, and of the proceedings of the International Urogynecological Association and International Continence Society from 2004 to 2006. Subjective measures of cure were analyzed, since only some articles used objective measures.

Eleven reports (4 full articles and 7 abstracts) were identified as being suitable for inclusion. A total of 1,261 women aged between 40 and 85 years were included across studies. Six trials compared TOT and five TVTO with TVT. In terms of cure, TVTO was slightly worse than and TOT was equal to TVT. The vaginal erosion rate was significantly higher in the TOT recipients and nonsignificantly lower in the TVTO recipients than among those who received TVT.

Only short-term data were available that did not clearly show notable advantages to using TOT and TVTO. The authors call for the initiation of long-term, well-designed, randomized, controlled trials and, in the interim, prospective collection of outcome data for audit.

Original article Latthe PM et al. (2007) Transobturator and retropubic tape procedures in stress urinary incontinence: a systematic review and meta-analysis of effectiveness and complications. BJOG 114: 522-531

\section{Collagen XXIII-a promising biomarker for prostate cancer metastasis}

Banyard and co-workers have previously reported a novel collagen, type XXIII, which is highly expressed in metastatic rat prostate cancer cells compared with poorly metastatic cell lines. Now, these researchers demonstrate that collagen XXIII has prognostic value in human prostate cancer.

Immunohistochemical staining for collagen XXIII was conducted on samples obtained from patients with prostate cancer who had undergone radical prostatectomy at the University Hospital Ulm, Germany, during the period 1986-2002. Only very low levels of collagen XXIII staining were detected in benign prostate epithelia. Collagen XXIII expression was considerably higher in primary prostate tumor samples than in benign samples, but the highest level of collagen XXIII expression was found in those with distant metastases. A retrospective study of 76 patients demonstrated that a high level of collagen XXIII staining was significantly associated with time to prostate-specific antigen (PSA) failure; patients with high levels of collagen XXIII had a median time to PSA failure of 8.1 months, compared with 5.0 years for those with low levels of this biomarker. Multivariate analysis revealed collagen XXIII to have prognostic value for PSA-specific disease recurrence, independent of tumor stage, Gleason score and preoperative PSA level.

On the basis of these results, and the finding that collagen XXIII can be detected in urine samples of patients with prostate cancer, the 